\title{
Alteration of the Fibrocartilaginous Nature of Scaffoldless Constructs Formed from Leporine Meniscus Cells and Chondrocytes through Manipulation of Culture and Processing Conditions
}

\author{
Daniel J. Huey Kyriacos A. Athanasiou \\ Department of Biomedical Engineering, University of California, Davis, Calif., USA
}

\section{Key Words}

Cartilage - Chondrocytes - Fibrocartilage - Meniscus .

Redifferentiation · Scaffoldless constructs · Tissue

engineering

\section{Abstract}

Articular cartilage and the menisci of the knee joint lack intrinsic repair capacity; thus, injuries to these tissues result in eventual osteoarthrotic changes to the joint. Tissue engineering offers the potential to replace damaged cartilage and mitigate long-term debilitating changes to the joint. In an attempt to enhance the ability of adult articular chondrocytes (ACs) and meniscus cells (MCs) to produce robust scaffoldless neocartilage, the effects of passage number, cryopreservation, and redifferentiation prior to construct formation were studied. By increasing passage number, smaller donor biopsies could be used to generate sufficient cells for tissue engineering and, in this study, no detrimental effects were observed when employing passage- 4 versus passage- 3 cells. Cryopreservation of cells would enable the generation of a cell bank thus reducing lead time and enhancing consistency of cell-based therapies. Interestingly, cryopreservation was shown to enhance the biomechanical properties of the resultant self-assembled constructs. With regard to redifferentiation prior to construct formation, aggregate redifferentiation was shown to enhance the biochemical and biomechanical properties of self-assembled constructs. By increasing passaging number, cryopreserving cells, and applying aggregate redifferentiation prior to neotissue formation, the utility of ACs and MCs in tissue engineering can be enhanced.

Copyright $\odot 2013$ S. Karger AG, Basel

\section{Abbreviations used in this paper}

AACR acellular and aproteinaceous central region

ACI autologous chondrocyte implantation

ACs articular chondrocytes

CM culture medium

ECM extracellular matrix

GAG glycosaminoglycan

MCs meniscus cells

P3 passage 3

P4 passage 4

TGF- $\beta 1 \quad$ transforming growth factor $\beta 1$

WW wet weight

\section{KARGER}

E-Mail karger@karger.com www.karger.com/cto
(C) 2013 S. Karger AG, Basel

$1422-6405 / 13 / 1975-0360 \$ 38.00 / 0$
Dr. Kyriacos A. Athanasiou

Department of Biomedical Engineering, University of California One Shields Avenue

Davis, CA 95616 (USA)

E-Mail athanasiou@ ucdavis.edu 


\section{Introduction}

Due to the knee's complex nature and the large forces transmitted through it, cartilages of the knee joint are commonly injured in sports, traumatic injuries, or due to age-related tissue degeneration. The two major cartilaginous structures of this joint, the knee meniscus and articular cartilage, are prone to injury because they are responsible for distributing and bearing the high loads that develop during bipedal activities. Due to the lack of blood supply to the inner portion of the meniscus and articular cartilage, the likelihood of sufficient intrinsic repair following injury is very low and permanent degenerative changes in these structures will occur [Athanasiou and Sanchez-Adams, 2009]. Thus, investigation of tissue engineering treatments aimed at regenerating native meniscal and articular cartilage tissues is of great interest.

Recently, scaffoldless techniques have emerged as viable options to engineer robust cartilage constructs [Park et al., 2006; Jubel et al., 2008; Cheuk et al., 2011]. These approaches mitigate the significant issues associated with scaffold use including toxicity of the material and its degradation products, stress-shielding, and matching degradation and tissue ingrowth rates [ $\mathrm{Hu}$ and Athanasiou, 2006]. One of these scaffoldless techniques, the self-assembly process, has been used to form cartilaginous tissues that mimic the biochemical and biomechanical properties of both the knee meniscus and articular cartilage [Hu and Athanasiou, 2006; Ofek et al., 2008; Huey et al., 2012]. This process employs a nonadherent, agarose well into which cells are seeded. These cells coalesce via $\mathrm{N}$ cadherin bonding and produce extracellular matrix (ECM) to form a robust tissue construct [Ofek et al., 2008] containing glycosaminoglycan (GAG) and collagen.

For any tissue engineering technique, a primary concern related to eventual clinical translatability is the selection of an appropriate cell source. For this decision, it is prudent to consider cell-based treatments that the FDA has deemed acceptable. For cartilage tissue engineering, autologous chondrocyte implantation (ACI) is the most relevant as it has been used for nearly 2 decades to treat focal cartilage defects. In the ACI technique, autologous, passaged and, most often, adult chondrocytes are injected under a flap that is secured over a cartilage defect to effect cartilage regeneration. In an effort to mimic this cell source while enabling future in vivo assessments in a small animal model, passaged, adult, leporine meniscus cells (MCs) and articular chondrocytes (ACs) were employed in this study.

The detrimental phenotypic shift that results from monolayer expansion of ACs and MCs can be modulated and partially mitigated via selection of appropriate expansion conditions. As ACs and MCs flatten and change their metabolic emphasis from matrix synthesis to cell proliferation, they lose the ability to synthesize cartilaginous matrix components (e.g. collagen type 2 and aggrecan) and take on a more fibrous phenotype [Darling and Athanasiou, 2005; Gunja and Athanasiou, 2007]. This shift makes engineering cartilaginous tissue with expanded cells challenging. Recently, researchers have made great strides in overcoming this issue by employing a chemically defined expansion medium and high seeding density [Mandl et al., 2004; Hendriks et al., 2006; Huey et al., 2012]. In particular, one study employed a chondrogenically tuned expansion protocol using a serum-free expansion medium containing insulin, dexamethasone, and basic fibroblast growth factor in combination with a high seeding density [Huey et al., 2012]. When applying this expansion protocol to adult leporine ACs, the resultant constructs contained higher GAG per wet weight (WW) and collagen type 2:collagen type 1 ratios compared to constructs formed with primary ACs or ACs expanded in a serum containing medium [Huey et al., 2012]. The effects of the aforementioned chemical agents have also been shown to be beneficial to the maintenance and redifferentiation capacity of MCs [Tumia and Johnstone, 2004; Adesida et al., 2012]. Thus, this study employed a chondrogenically tuned expansion protocol to both ACs and MCs.

In an effort to further enhance potential clinical utility of passaged ACs and MCs, three factors were tested that would either (1) decrease the lead time required for an allogenic cell-based approach, (2) decrease the biopsy size needed to obtain an adequate amount of cells for an autologous cell-based treatment, or (3) enhance the cartilaginous properties of tissue formed in any cell-based approach.

Cryopreservation of expanded ACs and MCs would allow for a large bank of allogenic cells to be generated, stored, and available for immediate usage; thus, lead time for the generation of neocartilage would be reduced. Although this relies on the ability to use allogeneic chondrocytes, in vitro assays and a prospective product [DeNovo ET (Zimmer, Warsaw, Ind., USA)] suggest that an allogenic cell source can be used without concerns of immune rejection [Adkisson et al., 2010]. While the effects of cryopreservation have been studied with primary ACs [Gan and Kandel, 2007; Seddighi et al., 2008; Lyu et al., 2010], there are few studies looking at the effects of cryopreservation of expanded ACs [Rendal-Vazquez et al., 2001; Martin et al., 2005] and none examining the effects of cryopreservation of primary or expanded MCs. It is necessary to de- 
termine the effects of cryopreservation on expanded cells as techniques may need to be adjusted to match the needs of expanded cells. Thus, this study examined the effects of cryopreservation on expanded rabbit ACs and MCs.

While the creation of a defect for cell harvest is a necessary part of autologous cell-based tissue engineering approaches, complications related to donor site morbidity result. In an effort to reduce the size of the biopsy, cells can be expanded to higher passages to obtain a greater number of cells from the same size biopsy. Typically, increasing the number of passages has been found to decrease the chondrogenic potential of chondrocytes [Darling and Athanasiou, 2005]. However, as new media formulation and expansion techniques are developed, the effects of passage number must be reevaluated. Thus, this study compared the usage of passage 3 (P3) and passage 4 (P4) ACs and MCs to determine if $\mathrm{P} 4$ cells can generate robust constructs.

Although expanded ACs and MCs redifferentiate in 3D culture, they will transiently produce fibrous ECM during the phenotypic shift from fibroblastic to cartilaginous [Mandl et al., 2004; Giannoni et al., 2005; Huey et al., 2012]. Previously, self-assembly of expanded cells has resulted in the formation of a central region within the construct that is devoid of living cells and matrix [Huey et al., 2012]. This structure is thought to arise due to the inability of a subset of the cells to adapt to the nonadherent conditions of self-assembly. Redifferentiation culture prior to construct formation may be able to simultaneously remove this adherence-dependent population and enhance the cartilaginous quality of constructs formed from the resulting cells. In this study, two commonly used techniques for chondrogenic redifferentiation, aggregate [Wolf et al., 2008] and pellet culture [Barbero et al., 2004; Giannoni et al., 2005], will be employed and compared to nonredifferentiated cells in terms of their ability to form robust constructs lacking an acellular and aproteinaceous central region (AACR).

This study will employ a full-factorial design with three factors: cryopreservation, passage number, and redifferentiation culture. Two levels of the cryopreservation factor will be studied: immediate use after expansion (noncryoperserved) or cryopreserved for 1 month. Two levels of the passage number factor will be examined: P3 or $\mathrm{P} 4$. Three levels of the redifferentiation factor will be studied: no redifferentiation, 1-week aggregate culture, or 1 -week pellet culture. Constructs formed with either primary MCs or ACs will serve as controls. The three hypotheses that will be investigated in this study follow: (1) cryopreservation of expanded ACs and MCs will not sig- nificantly alter resulting construct properties; (2) as a result of chondrogenically tuned expansion, $\mathrm{P} 3$ and P4 ACs or MCs will create constructs of similar biomechanical and biochemical quality, and (3) redifferentiation culture, particularly aggregate culture due to its similarity with self-assembly, will be beneficial to the biochemical and biomechanical properties of resulting tissue constructs.

\section{Materials and Methods}

Chondrocyte Isolation and Expansion

Knee joints of skeletally mature New Zealand White rabbits (Heaton Rabbitry, Huntsville, Tex., USA) were obtained within $8 \mathrm{~h}$ of sacrifice. Approval of the Institutional Animal Care and Use Committee was not required because the rabbits were euthanized by the provider as part of a routine herd cull. Articular cartilage from both tibial and femoral surfaces and meniscal fibrocartilage were sterilely dissected. These tissues were minced into $\sim 1-\mathrm{mm}$ pieces and digested in $0.2 \%$ collagenase type 2 (Worthington, Lakewood, N.J., USA) in chemically defined chondrogenic culture medium (CM; DMEM with 4.5 g/l glucose and GlutaMAX; Invitrogen, Carlsbad, Calif., USA), 100 nM dexamethasone, 1\% Fungizone/penicillin/streptomycin (Fisher Scientific, Pittsburgh, Pa., USA), 1\% ITS+ premix (BD Biosciences, Bedford, Mass., USA), 50 $\mathrm{mg} / \mathrm{ml}$ ascorbate-2-phosphate (Sigma-Aldrich, St. Louis, Mo., USA), $40 \mathrm{mg} / \mathrm{ml} \mathrm{L}$-proline (Sigma-Aldrich), and $100 \mathrm{mg} / \mathrm{ml}$ sodium pyruvate (Fisher Scientific). Following overnight digestion, cells were isolated via sequential centrifugation and PBS dilution. Finally, cells were resuspended in freezing media (CM with $20 \%$ fetal bovine serum; Gemini Bio-Products, Woodland, Calif., USA) and $10 \%$ DMSO (Sigma-Aldrich). Vials containing $1 \mathrm{ml}$ of freezing media containing 10 million cells were frozen at $1^{\circ} \mathrm{C} / \mathrm{min}$ to $-80^{\circ} \mathrm{C}$ and then placed into liquid nitrogen cryostorage.

The protocols employed to expand both ACs and MCs are based on previous work [Huey et al., 2012]. Briefly, primary ACs and MCs were thawed and seeded onto T-225 flasks at a density of $2.5 \times 10^{4}$ cells $/ \mathrm{cm}^{2}$ in CM supplemented with $5 \mathrm{ng} / \mathrm{ml}$ basic fibroblast growth factor (PeproTech, Rocky Hill, N.J., USA). During the first $24 \mathrm{~h}$ following seeding or a passaging event, it was necessary to add $10 \%$ fetal bovine serum to allow adequate cell adhesion. Besides this initial 24-hour period, all cells were expanded in CM with basic fibroblast growth factor, which was changed every other day. Following the previously described protocol, ACs and MCs were allowed to expand in monolayer culture for 4 days after confluence had been reached. At this point, cells were lifted from the flask using $0.05 \%$ trypsin/EDTA (Invitrogen) and digested with $0.2 \%(\mathrm{w} / \mathrm{v})$ collagenase $(220 \mathrm{U} / \mathrm{mg}$; Worthington) to obtain a single-cell suspension. Cell solutions were combined with trypan blue and counted on a hemocytometer. Cells were expanded to P3 or P4 under these conditions and then either cryopreserved for 4 weeks before use or used immediately for construct formation with or without redifferentiation. Cells were cryopreserved at a cell density of 10 million cells per milliliter and $1 \mathrm{ml}$ of this solution was added to each vial. Vials were cooled at $1^{\circ} \mathrm{C} / \mathrm{min}$ to $-80^{\circ} \mathrm{C}$ and then transferred to liquid nitrogen. 


\section{$A C$ and MC Redifferentiation}

Two types of redifferentiation culture were employed: pellet culture or aggregate culture. Cells from each of the 8 distinct groups (two cell types, two passage numbers, and either cryopreserved or used immediately after expansion) were either subjected to one of the two redifferentiation cultures or immediately used for construct formation. Aggregate culture involved seeding 700,000 cells $/ \mathrm{cm}^{2}$ onto a $2 \%$ agarose (Fisher Scientific)-coated Petri dish in $\mathrm{CM}$ with $10 \mathrm{ng} / \mathrm{ml}$ transforming growth factor (TGF)- $\beta_{1}$ (PeproTech). Pellet culture involved centrifugation at $500 \mathrm{~g}$ of 250,000 cells in each well of a V-bottom 96-well plate and culturing in CM with $10 \mathrm{ng} / \mathrm{ml} \mathrm{TGF}-\beta_{1}$. The duration of redifferentiation culture was 1 week for both of the techniques and media were changed every other day. Following redifferentiation culture, aggregates and pellets were digested for $1 \mathrm{~h}$ in $0.05 \%$ trypsin/EDTA (Invitrogen) followed by $1 \mathrm{~h}$ in $0.2 \%$ collagenase (Worthington) and filtration through a $70-\mu \mathrm{m}$ mesh to obtain a cell solution.

\section{Construct Seeding}

Cells from the 24 distinct groups plus control primary ACs and MCs were used to create tissue constructs by placing 2 million cells into a 3-mm-diameter agarose well. To create agarose wells, $2 \%$ molten agarose was placed into a well of a 6 -well plate and a wellmaker consisting of multiple silicone posts $3 \mathrm{~mm}$ in diameter and $1 \mathrm{~cm}$ tall was plunged into the agarose. After $1 \mathrm{~h}$, when the agarose had set, the well-maker was removed to create an agarose cylinder containing multiple wells ( $3 \mathrm{~mm}$ in diameter and $1 \mathrm{~cm}$ deep). This agarose cylinder was allowed to equilibrate in CM for 1 week prior to construct seeding. Medium was changed every other day for the duration of the 4-week construct culture period and constructs remained within the agarose wells for the duration of the study. After 4 weeks, constructs were removed from the culture to perform gross morphological, histological, biochemical, and biomechanical assessments.

\section{Gross Morphology and Histology}

After removal from the culture, constructs were photographed to determine diameter and height. Constructs were then bisected at the midpoint of the height of the construct. Any liquid or pastelike material present within the AACR was removed and the halves were photographed again to enable quantification of the percentage of the cross-sectional area occupied by the AACR. Then both halves were weighed to obtain WW. If available, two entire constructs were used for histological examination but when the total number of constructs in a group precluded this, portions of at least two constructs were used. Histological samples were snap-frozen in HistoPrep (Fisher Scientific) and sectioned to $14 \mu \mathrm{m}$. Safranin$\mathrm{O} /$ fast green staining allowed the visualization of GAG distribution. Picrosirius red staining allowed the visualization of collagen distribution.

\section{Quantitative Biochemistry}

Following lyophilization, the dry weight of biochemical samples was determined. Collagen, GAG, DNA, and collagen type 1 and 2 content was quantitatively measured through various assays. First, constructs $(n=6)$ were solubilized by digestion in a $1.1 \mathrm{mg} /$ $\mathrm{ml}$ pepsin (Sigma) solution at $4^{\circ} \mathrm{C}$ and a subsequent 2-day digestion in a $100 \mu \mathrm{g} / \mathrm{ml}$ elastase (Sigma) solution. Following digestion, the Blyscan GAG assay (Biocolor, Carrickfergus, UK), a modified colorometric hydroxyproline assay with collagen standards as con- trols [Almarza and Athanasiou, 2004], the PicoGreen (Invitrogen) assay for DNA content, and enzyme-linked immunosorbent assays for collagens 1 and 2 were performed. For collagen type 1 and 2 quantification, a sandwich ELISA technique was used. For collagen type 2, Chondrex (Redmond, Wash., USA) antibodies and standards were used. For collagen type 1, US Biological (Boston, Mass., USA) antibodies and Chondrex standards were used. DNA content data from the PicoGreen assay were converted to cell numbers using a conversion factor of $7.7 \mathrm{pg}$ DNA per cell [Kim et al., 1988].

\section{Unconfined Compression Testing}

Images of cylindrical construct portions designated for compression testing were taken to accurately determine the diameter of the test specimen. The unconfined compression test employed for this study has been described in detail previously [Huey and Athanasiou, 2011]. Briefly, test specimens were placed into a PBS bath and compressed to 10 and $20 \%$ of their initial height using an Instron 5565 (Norwood, Mass., USA). Each strain increment was held for $10 \mathrm{~min}$. In conjunction with a custom program, the curve fitting tools on Matlab (Mathworks, Natick, Mass., USA) were used to determine viscoelastic compressive properties (relaxation modulus, instantaneous modulus, and coefficient of viscosity) as previously described [Huey et al., 2012]. Portions of 6 constructs from each group were assessed for compressive biomechanical properties.

\section{Tensile Testing}

Tensile samples were cut into a dog bone shape and photographed to ensure the accurate determination of geometric properties. Immediately prior to testing, samples were glued onto a strip of paper spanning a precisely measured gap. The strip of paper was grasped in the clamps of an Instron 5565 and then the strip was cut to allow the load to only act on the sample. Specimens were elongated at $1 \%$ of the gauge length per second until failure. Geometric, load, and elongation data were processed using a custom program to isolate the linear region and determine the Young modulus of the material.

\section{Statistics}

A three-factor ANOVA was used to examine the results obtained from biochemical and biomechanical testing with $\mathrm{n}=6$. All results were blocked by cell type. The three factors and corresponding levels follow: passage number (P3, P4), cryostorage after expansion (yes, no), and redifferentiation before self-assembly (no, pellet, aggregate). Tukey's post hoc test was used, when needed (main effects test: $\mathrm{p}<0.05)$, to determine significant differences among levels of a factor and among all groups $(\mathrm{p}<0.05)$. In figures and tables, significant differences are present when a common letter is not shared.

\section{Results}

\section{Cell Expansion and Redifferentiation}

Both ACs and MCs were able to proliferate in the expansion conditions provided. AC cell morphology underwent a shift from a rounded to polygonal, cobblestone 


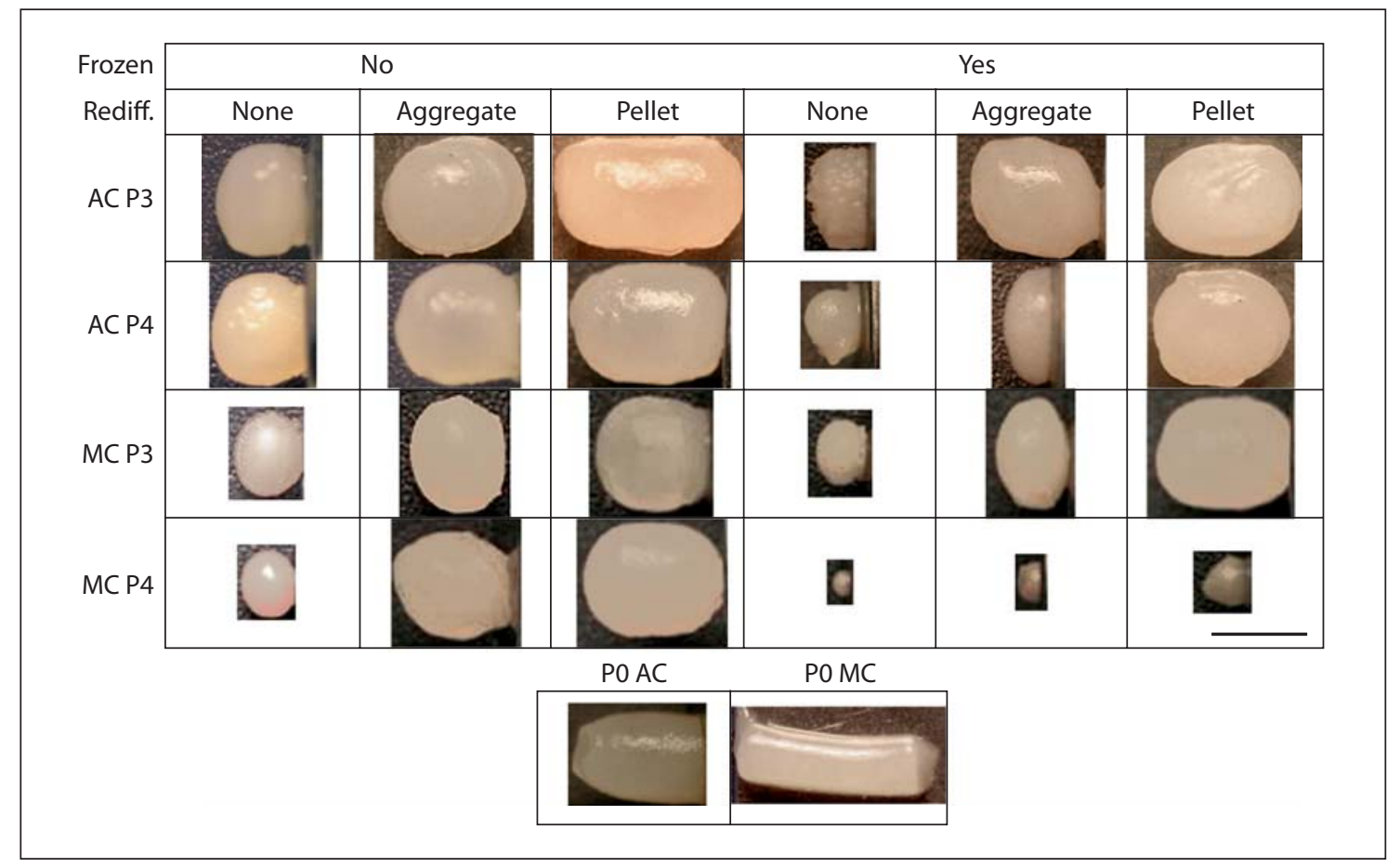

Fig. 1. Gross morphology: representative images of constructs from each of the 24 experimental groups and 2 primary cell controls. Bar $=3 \mathrm{~mm}$.

Table 1. Explanation of construct group naming abbreviations (redifferentiation: none; Aggr. = aggregate, and pellet)

\begin{tabular}{|c|c|c|c|c|c|c|c|c|c|c|c|c|}
\hline & \multicolumn{5}{|c|}{ Not frozen } & \multicolumn{7}{|l|}{ Frozen } \\
\hline & none & Aggr. & pellet & none & Aggr. pellet & none & Aggr. & pellet & none & Aggr. & pellet & $\begin{array}{l}0 \\
\text { NA }\end{array}$ \\
\hline AC constructs & $3 \mathrm{ANN}$ & 3ANA & 3ANP & $4 \mathrm{ANN}$ & 4ANA $4 \mathrm{ANP}$ & $3 \mathrm{AYN}$ & 3AYA & 3AYP & $4 \mathrm{AYN}$ & 4AYA & 4AYP & $0 \mathrm{~A}$ \\
\hline MC constructs & $3 \mathrm{MNN}$ & $3 \mathrm{MNA}$ & $3 \mathrm{MNP}$ & $4 \mathrm{MNN}$ & 4MNA 4MNP & $3 \mathrm{MYN}$ & 3MYA & 3MYP & $4 \mathrm{MYN}$ & 4MYA & 4MYP & $0 \mathrm{M}$ \\
\hline
\end{tabular}

shape as passage number increased. A similar change in cell morphology was also noted for MCs; however, these cells began, and remained, more fibroblastic. ACs reached P3 after 19 days of culture and underwent a 65-fold increase in cell number. Expansion to P4 took an additional 5 days and resulted in a further 4 -fold increase in cell number. MCs required 17 days of culture to reach $\mathrm{P} 3$ and resulted in a 57-fold increase in cell number. To reach $\mathrm{P} 4$, MCs required another 4 days of culture and resulted in a 3.5 -fold increase in cell number. Regardless of the cell type, cryopreservation, or passage number, redifferentiation cultures appeared similar. The aggregate culture system initially formed round cell clumps ranging from 10 to hundreds of cells. Over time, these aggregates coalesced to form larger aggregates and networks of aggregates connected at their edges. Pellet culture consistently formed spherical pellets approximately $1 \mathrm{~mm}$ in diameter.

\section{Gross Morphology and Histology}

The group abbreviations used for this study are shown in table 1. Gross morphological images are shown in figure 1 . In addition, mean values for geometric properties, WW, and percent hydration can be found in table 2 and the statistical effects of the various treatments on these properties can be found in table 3. Upon bisection and histological examination, a majority of the constructs ex- 


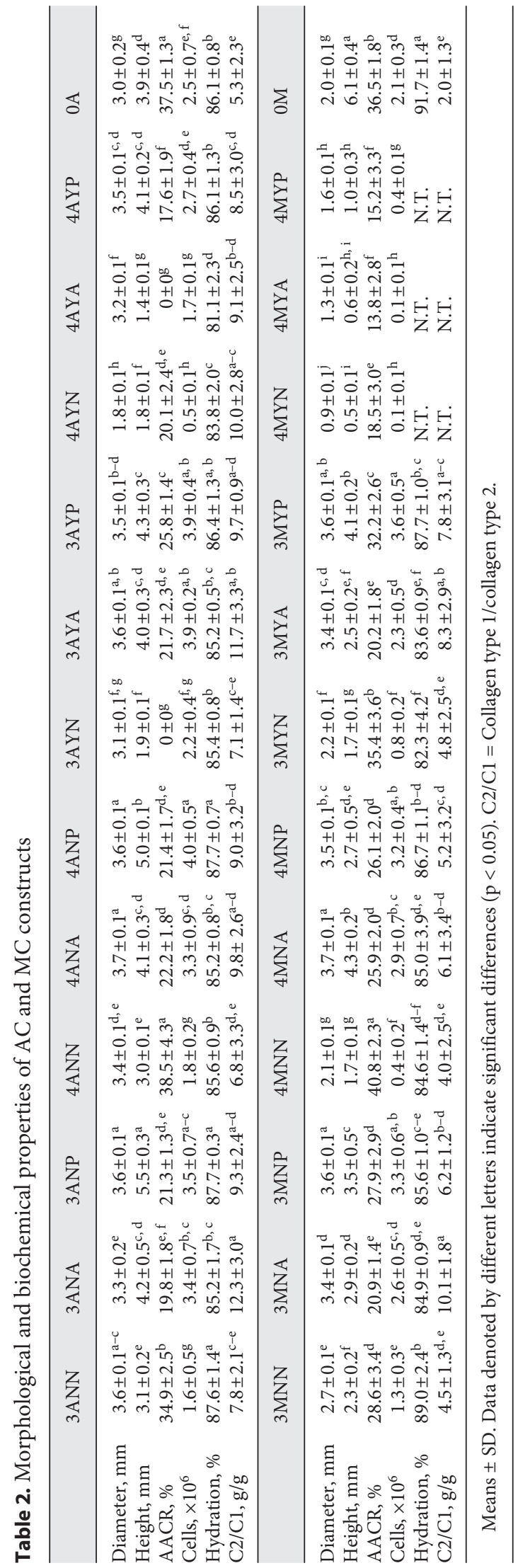

Enhancing Self-Assembled Leporine ACs and MCs
Table 3. Statistically significant effects of the levels of the three factors on morphological, biochemical and biomechanical properties

\begin{tabular}{|c|c|c|c|c|c|c|c|}
\hline \multirow{2}{*}{$\begin{array}{l}\text { Factor } \\
\text { Level }\end{array}$} & \multicolumn{2}{|c|}{ Frozen } & \multicolumn{2}{|c|}{ Passage } & \multicolumn{3}{|c|}{ Rediff. } \\
\hline & no & yes & 3 & 4 & $\mathrm{~N}$ & A & $\mathrm{P}$ \\
\hline \multicolumn{8}{|l|}{ AC constructs } \\
\hline Diameter & $\mathrm{A}$ & B & $\mathrm{A}$ & B & $\mathrm{B}$ & A & A \\
\hline Height & A & B & A & B & $\mathrm{C}$ & B & A \\
\hline AACR & $\mathrm{A}$ & B & $\mathrm{A}$ & $\mathrm{A}$ & $\mathrm{A}$ & B & $\mathrm{A}$ \\
\hline Cell & $\mathrm{A}$ & B & $\mathrm{A}$ & B & $\mathrm{C}$ & $\mathrm{B}$ & A \\
\hline Hydration & A & B & A & B & B & $\mathrm{C}$ & A \\
\hline $\mathrm{C} 2 / \mathrm{C} 1$ & $\mathrm{~A}$ & A & $\mathrm{A}$ & $\mathrm{A}$ & B & A & B \\
\hline GAG/WW & $\mathrm{B}$ & A & B & $\mathrm{A}$ & $\mathrm{B}$ & A & $\mathrm{C}$ \\
\hline Collagen/WW & B & A & $\mathrm{A}$ & $\mathrm{A}$ & $\mathrm{B}$ & A & B \\
\hline $\mathrm{E}_{\mathrm{R}}$ & B & A & $\mathrm{B}$ & A & B & A & B \\
\hline $\mathrm{E}_{\infty}$ & $\mathrm{B}$ & A & B & $\mathrm{A}$ & $\mathrm{B}$ & A & B \\
\hline Tensile $\mathrm{E}_{\mathrm{Y}}$ & $\mathrm{B}$ & A & $\mathrm{A}$ & $\mathrm{A}$ & $\mathrm{B}$ & A & B \\
\hline \multicolumn{8}{|l|}{ MC constructs } \\
\hline Diameter & A & B & A & B & B & A & A \\
\hline Height & A & A & $\mathrm{A}$ & $\mathrm{A}$ & B & A & A \\
\hline AACR & $\mathrm{A}$ & B & $\mathrm{A}$ & $\mathrm{B}$ & $\mathrm{A}$ & $\mathrm{C}$ & B \\
\hline Cell & A & A & A & A & $\mathrm{C}$ & B & A \\
\hline Hydration & $\mathrm{A}$ & B & $\mathrm{A}$ & A & $\mathrm{AB}$ & B & A \\
\hline $\mathrm{C} 2 / \mathrm{C} 1$ & $\mathrm{~A}$ & A & $\mathrm{A}$ & $\mathrm{B}$ & $\mathrm{C}$ & A & B \\
\hline GAG/WW & B & A & $\mathrm{B}$ & A & $\mathrm{B}$ & A & B \\
\hline Collagen/WW & $\mathrm{B}$ & A & $\mathrm{A}$ & A & $\mathrm{A}$ & A & B \\
\hline $\mathrm{E}_{\mathrm{R}}$ & $\mathrm{B}$ & A & A & A & $\mathrm{B}$ & A & $\mathrm{C}$ \\
\hline $\mathrm{E}_{\infty}$ & $\mathrm{B}$ & $\mathrm{A}$ & $\mathrm{A}$ & $\mathrm{A}$ & $\mathrm{C}$ & A & B \\
\hline Tensile $E_{Y}$ & B & $\mathrm{A}$ & $\mathrm{A}$ & $\mathrm{A}$ & A & A & B \\
\hline
\end{tabular}

The effect of a level denoted with an A is significantly greater than $B$ and the effect of $B$ is significantly greater than $C$, when necessary. $\mathrm{C} 2 / \mathrm{C} 1=$ Collagen type $1 /$ collagen type $2 ; \mathrm{E}_{\mathrm{R}}=$ relaxation modulus; $\mathrm{E}_{\infty}=$ instantaneous modulus; $\mathrm{E}_{\mathrm{Y}}=$ Young's modulus; for Rediff. factors: $\mathrm{N}=$ none; $\mathrm{A}=$ aggregate; $\mathrm{P}=$ pellet.

hibited an AACR. However, in 4AYA this structure was not present and in 4MYA the size of the AACR was significantly reduced. The percentage of the construct crosssection that was occupied by the AACR is shown in table 2 and was shown to decrease with freezing and redifferentiation (table 3; fig. 2). In constructs that did not undergo a redifferentiation step, the substance within the AACR was beige in color, had a paste-like texture, and, upon trypan blue staining, was shown to be heavily populated with dead cells. When redifferentiation was applied, the AACR was smaller, filled with a clear fluid, and very few dead cells were observed after trypan blue staining. AACR percentage ranged from 0 (4AYA, $3 \mathrm{AYN}$ ) to $38.5 \%$ (4ANN) for AC constructs and from 13.8 (4MYA) to $40.8 \%(4 \mathrm{MNN})$ for $\mathrm{MC}$ constructs. Although, constructs were confined in $3-\mathrm{mm}$ wells, the $4 \mathrm{ANA}$ and the $4 \mathrm{MNA}$ 


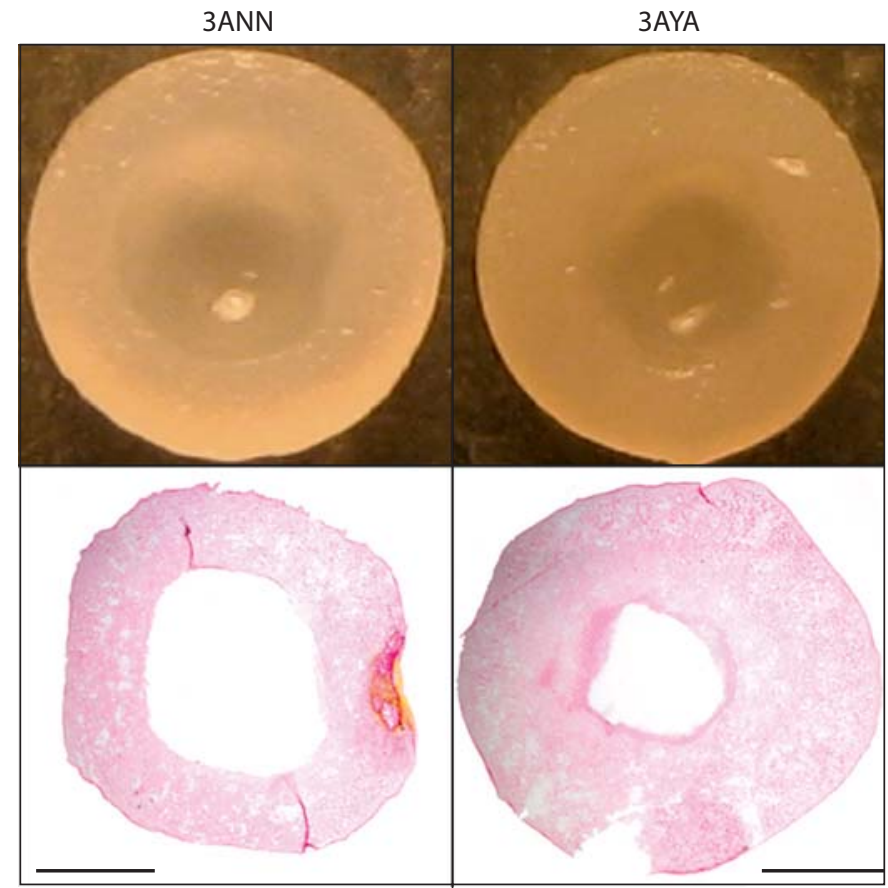

Fig. 2. AACR: morphological images and picrosirius red staining of bisected constructs taken illustrate the AACR and indicate that cryopreservation and aggregate redifferentiation reduced the size of the AACR. Bar $=1 \mathrm{~mm}$.

groups reached $3.7 \mathrm{~mm}$ in diameter by radially deforming the agarose wells, which was the maximum for $\mathrm{AC}$ and $\mathrm{MC}$ constructs, respectively. For AC constructs, height ranged from $1.4(4 \mathrm{AYN})$ to $5.5 \mathrm{~mm}(3 \mathrm{ANP})$ and for $\mathrm{MC}$ constructs from $0.5(4 \mathrm{MYN})$ to $6.1 \mathrm{~mm}(0 \mathrm{M})$. Construct hydration varied from 81.1 (4AYA) to $87.7 \%$ (3ANP, $4 \mathrm{ANP}$ ) for AC constructs and from 82.3 (3MYN) to $91.7 \%$ (0M) for MC constructs.

Histological staining for collagen and GAG through picrosirius red and safranin $\mathrm{O}$ dyes revealed nothing remarkable regarding protein distribution. Differences in intensity follow the trends apparent in the quantitative biochemical analysis.

\section{Biochemistry}

Results of the biochemical assessments for collagen and GAG normalized to WW are shown in figure 3. Results for cell number and collagen 2 normalized to collagen 1 are shown in table 2. The effects of the levels of the various factors are shown in table 3. For AC constructs, collagen per WW ranged from 3.5 (3ANP) to $12.1 \%$ (4AYA). For MC constructs, collagen per WW ranged from 4.9 (4MNP) to $14.6 \%$ (3MYA). GAG per WW var- ied from $1.7(0 \mathrm{~A})$ to $8.3 \%$ (4AYA) for AC constructs and from $0.3(0 \mathrm{M})$ to $5.9 \%$ (3MYA) for MC constructs. Although all constructs were seeded with 2 million cells, final cell numbers ranged from 0.5 (4AYN) to 4 million (4ANP) for AC constructs and 0.1 (4MYN, 4MYA) to 3.6 million (4MNP) for MC constructs. Collagen 2 was more abundant than collagen 1 in all constructs, as illustrated by the collagen $2 /$ collagen 1 ratio that ranged from 5.3 $(0 \mathrm{~A})$ to $12.3(3 \mathrm{ANP})$ for $\mathrm{AC}$ constructs and $2.0(0 \mathrm{M})$ to 10.1 (3MNA) for MC constructs.

\section{Biomechanics}

The compressive relaxation modulus, compressive instantaneous modulus, and the tensile Young modulus were determined and are displayed in figure 4 . The effects of the levels of the various factors on these properties are found in table 3 . The relaxation modulus varied from 88 (0A) to $358 \mathrm{kPa}$ (4AYA) for AC constructs and from 37 $(0 \mathrm{M})$ to $330 \mathrm{kPa}$ (3MYA) for MC constructs. The instantaneous modulus ranged from 649 (3ANN) to $2,057 \mathrm{kPa}$ (4AYA) for AC constructs and from $143(0 \mathrm{M})$ to $1,969 \mathrm{kPa}$ (3MYA) for MC constructs. The tensile modulus varied from $164(0 \mathrm{~A})$ to $907 \mathrm{kPa}$ (4AYA) for AC constructs and from $106(0 \mathrm{M})$ to $1,271 \mathrm{kPa}(3 \mathrm{MYA})$ for MC constructs.

\section{Discussion}

This study assessed (1) the effects of redifferentiation of expanded ACs and MCs prior to construct formation, (2) the effects of cryopreservation of expanded ACs and MCs on biochemical and biomechanical properties of resulting constructs, and (3) the effects of passage in a chondrogenic expansion protocol. The first hypothesis of this study was that use of $\mathrm{P} 3$ and $\mathrm{P} 4$ cells would result in tissue of a similar quality. This hypothesis was proven through analysis of the values obtained from biochemical and biomechanical assessments. These determined that properties were either unchanged or significantly increased by employing $\mathrm{P} 4$ cells. The next hypothesis was that cryopreservation would not significantly affect the biochemical or biomechanical properties of resulting constructs. This hypothesis was disproven in a counterintuitive manner as cryopreservation enhanced collagen/WW, GAG/ $\mathrm{WW}$, and all biomechanical properties of both $\mathrm{AC}$ and $\mathrm{MC}$ constructs. The final hypothesis regarding the benefits of redifferentiation culture prior to self-assembly was proven in the case of aggregate culture as evidenced by increased GAG/WW, collagen type 2:collagen type 1 ratio, and compressive properties. 
Fig. 3. Biochemical properties. a Collagen/ WW. b GAG/WW. Significant differences $(\mathrm{p}<0.05)$ exist between groups that do not contain the same letter. $\mathrm{N}=\mathrm{No} ; \mathrm{Y}=$ yes.

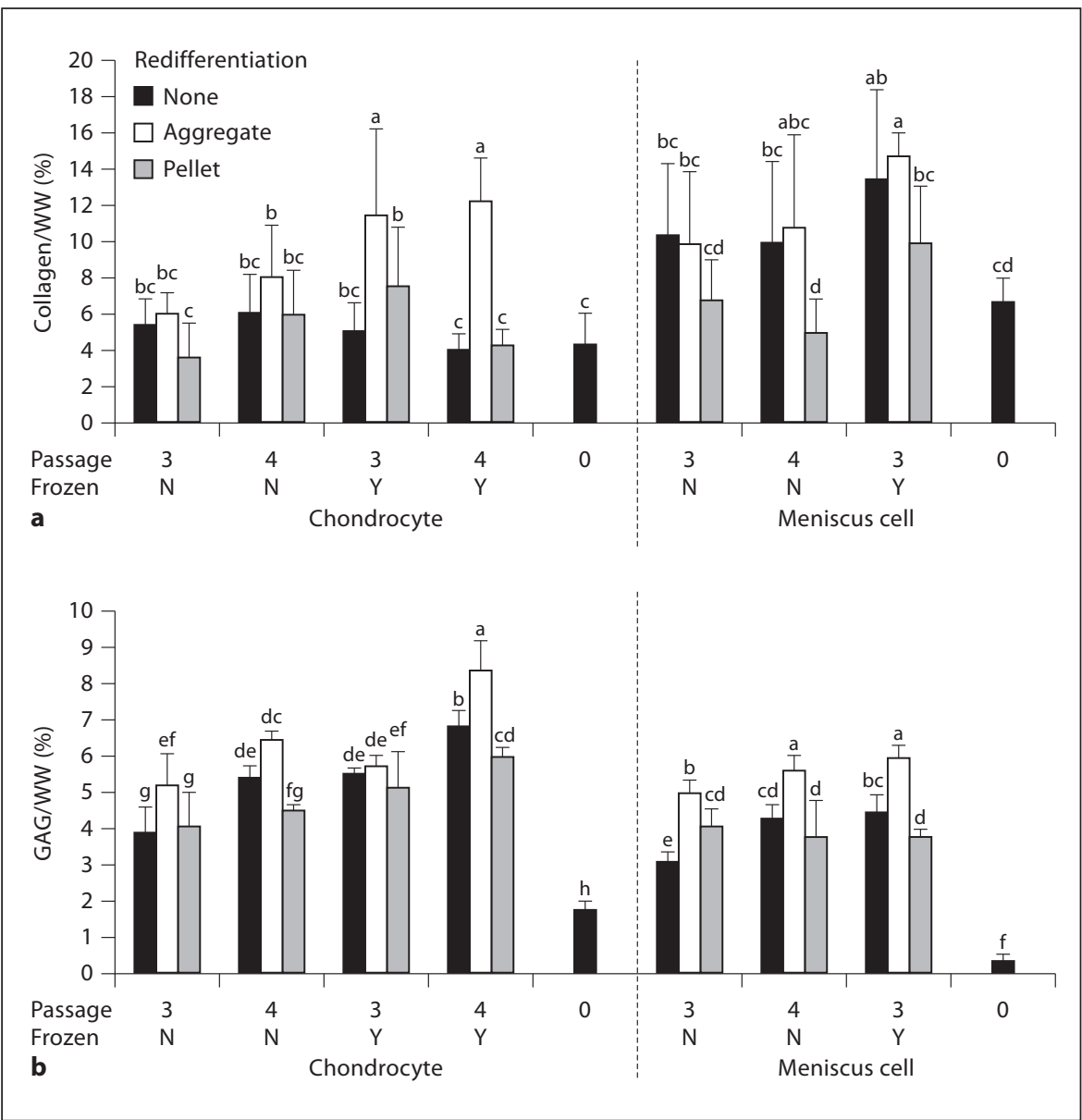

Previous studies have identified the detrimental effects of expansion in serum-containing media for both ACs and MCs [Darling and Athanasiou, 2005; Gunja and Athanasiou, 2007]. Contrary to those studies, this study indicated that expansion to P4 compared to P3 was not phenotypically disadvantageous. GAG/WW was shown to be increased in P4 ACs and MCs over their P3 counterparts. Due to passage number, the ratio of collagen type 2:collagen type 1 was unaffected in AC constructs and the tensile modulus was unaffected in both $\mathrm{AC}$ and MC constructs. The discrepancies between the results of this study and those previously conducted are likely due to the difference in the expansion protocol used. Similar to a previous research [Huey et al., 2012], this study showed that chondrogenically tuned expansion was able to enhance the cartilage-forming capability of primary cells. It is hypothesized that the poor performance of the primary cells is due to a decrease in SOX-9 expression associated with aging [Hidaka et al., 2006; Huey et al., 2012].
Exposure to chondrogenic factors known to enhance SOX-9 expression may account for the increase in the chondrogenic potential observed with expanded adult cells [Barbero et al., 2004; Malpeli et al., 2004; Giannoni et al., 2005]. Thus, it is possible that the full benefits of chondrogenically tuned expansion are not realized until higher passages.

While the effects of cryopreservation on primary ACs have been investigated, there is a dearth of studies examining its effects on passaged ACs and MCs. In this study, cryopreservation was shown to significantly enhance the biochemical and biomechanical properties of both AC and MC constructs. This is an intriguing result as cryopreservation had previously been shown to effect viability and not phenotype [Rendal-Vazquez et al., 2001; Lyu et al., 2010]. This study does agree with previous studies by showing that the cell phenotype was unaffected by cryopreservation as evidenced by an unchanged collagen type 2:collagen type 1 ratio. Thus, cell death related to 
Fig. 4. Biomechanical properties. a Compressive relaxation modulus. b Compressive instantaneous modulus. c Tensile Young modulus. Significant differences $(\mathrm{p}<0.05)$ exist between groups that do not contain the same letter. $\mathrm{N}=\mathrm{No} ; \mathrm{Y}=$ yes.

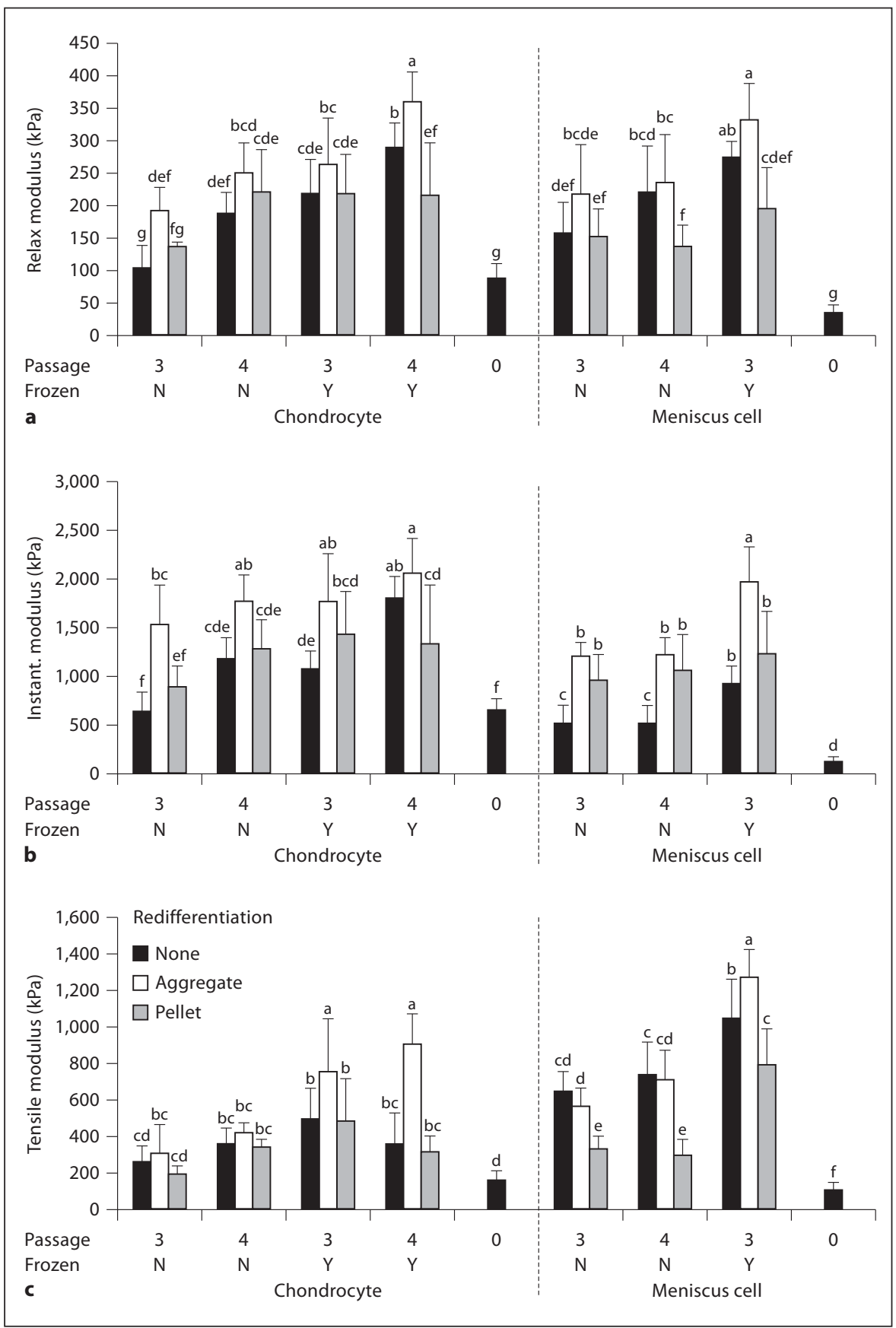

cryopreservation was likely a major contributor to the enhancement of construct properties. A potential explanation for this finding is that a subset of the inhomogenous cell population resulting from monolayer expansion was both more sensitive to cryopreservation and responsible for the more expansive construct growth observed when using noncryopreserved cells. Regardless, cryopreserva- tion altered the population of cells such that resultant constructs had decreased hydration and geometric properties which, when coupled with increases in GAG/WW and collagen/WW, suggests that cryopreservation increased cellular contractile tendencies. Overall, these results show that cryogenic storage of expanded ACs and MCs does not detrimentally affect the properties of resul- 
tant constructs, thus enabling banking of these cells to shorten the lead time in generating tissue-engineered cartilaginous constructs.

A redifferentiation phase was employed prior to construct formation for two distinct reasons. First, to enhance the cartilaginous qualities of constructs by decreasing the transient accumulation of fibrous ECM created during $3 \mathrm{D}$ culture-induced redifferentiation of expanded cells. The second reason is to reduce the size of the AACR by eliminating cells that were not capable of survival in the $3 \mathrm{D}$ environment presented by the self-assembly process. Pellet culture prior to self-assembly resulted in constructs with enhanced cellularity, increased hydration, and increased geometric properties compared to constructs formed following aggregate culture. Although pellet redifferentiation resulted in the generation of a large amount of tissue, the functional properties were not improved. This is likely due to the increase in construct hydration and concomitant decrease in ECM density and indicates undesired appositional construct growth [Asanbaeva et al., 2007]. Aggregate culture was more beneficial than either no redifferentiation or pellet redifferentiation in terms of the biochemical and biomechanical properties of AC constructs. With regard to MC constructs, aggregate redifferentiation resulted in constructs with significantly improved biochemical and biomechanical properties compared to pellet redifferentiation. Compared to no redifferentiation, aggregate redifferentiation significantly enhanced all metrics except collagen/WW and the tensile modulus where the effect of either level was equal. The higher degree of success associated with aggregate preculture may be due to the similarities between aggregate formation and self-assembly. Both of these processes rely upon the cells binding through $\mathrm{N}$-cadherins to create a 3D structure [Steinberg, 2007; Ofek et al., 2008]; therefore, aggregate redifferentiation may have been more efficient at eliminating cells that were unlikely to self-assemble. By decreasing hydration, increasing or maintaining ECM production, and eliminating cells not capable of self-assembly, aggregate preculture resulted in the formation of self-assembled constructs that possessed a smaller AACR and higher functional properties.

For both meniscal and articular cartilage, biomechanical functionality is critical for the success of a tissue replacement strategy. Of the AC constructs created in this study, the treatment combination 4AYA resulted in constructs with the highest biomechanical properties. For this group, increases of $200 \%$ in the instantaneous modulus, $240 \%$ in the relaxation modulus, and $240 \%$ in the tensile modulus were obtained compared to $3 \mathrm{ANN}$ treat-

Enhancing Self-Assembled Leporine ACs and MCs ment. In addition, the 4AYA had significantly higher biochemical and biomechanical properties compared to primary AC construct controls. For MC constructs, 3MYA treatment resulted in the highest biomechanical properties with increases of $110 \%$ in relaxation modulus, $277 \%$ in instantaneous modulus, and $95 \%$ in tensile modulus compared to $3 \mathrm{MNN}$ treatment. All biochemical and biomechanical properties were significantly higher for the 3MYA group compared to the primary MC construct control. These results show that with the proper selection of passage number, usage of cryopreservation, and application of redifferentiation culture, the functional properties of constructs formed from chondrogenically expanded cells can be enhanced over those of primary cell constructs.

Compared to native articular cartilage, the 4AYA treatment produced constructs that slightly exceeded native GAG/WW, had half the amount of collagen/WW, reached $50 \%$ of the relaxation modulus, had a greater instantaneous modulus, and were an order of magnitude lower in tensile modulus [Wei et al., 1998; Athanasiou et al., 2009]. For MC constructs compared to native meniscal tissue, GAG/WW was slightly higher, $66 \%$ of collagen/ WW was recapitulated, both relaxation and instantaneous moduli equaled, and construct tensile properties were on par with the tensile modulus of native tissue in the radial direction but was significantly lower than native tissue in the circumferential direction [Chia and Hull, 2008; Athanasiou and Sanchez-Adams, 2009]. However, it is difficult to compare the tensile moduli of either the $\mathrm{AC}$ or MC constructs created in this study to native tissue because the curved geometry of tensile specimens would have resulted in tensile moduli lower than the true value. For both AC and MC constructs, the main deficiency is collagen content and tensile properties. Recent attempts employing the self-assembly process with other cell types have shown the ability to greatly enhance collagen content and tensile properties through the application of chondroitinase ABC and TGF- $\beta_{1}$ [Huey and Athanasiou, 2011]. In the future, the applicability of these chemical agents to the constructs formed by the treatments identified in this study should be examined.

Cartilage biopsies performed to obtain the cells required for ACI weigh approximately $250 \mathrm{mg}$ [Mayhew et al., 1998]. Chondrocytes are present in adult cartilage at a density of about 4.5 million cells per gram [Barbero et al., 2004]. Thus, the current standard for cartilage biopsies prior to a cell-based therapy yields approximately 1 million cells. Based on the protocol in this study that prevented AACR formation (4AYA), 1 million primary cells 
would create enough tissue construct to cover $10.5 \mathrm{~cm}^{2}$. Even at $\mathrm{P} 3$, constructs covering $2.6 \mathrm{~cm}^{2}$ could be created. These results show that the cartilage-forming procedure described in this study is clinically attractive not only with respect to the quality of the neocartilage but also regarding cell numbers.

This study assessed the effects of three factors (passage number, cryopreservation, and redifferentiation) applied prior to construct formation on the cartilaginous quality of the resultant constructs. For ACs, increasing the passage number from P3 to P4 was shown to enhance compressive properties of resultant constructs, while passage number did not alter biomechanical properties for MCs. Warranting additional studies, cryopreservation resulted in statistically significant increases in collagen and GAG contents and biomechanical properties. Finally, use of aggregate culture prior to construct formation to redifferentiate the ACs and MCs resulted in self-assembled constructs with superior biomechanical properties. Overall, this study was able to improve the utility of ACs and MCs in tissue engineering by not only determining methods to enhance cartilaginous properties of resultant constructs but also by enabling the use of cryopreservation and higher-passage cells without concerns of deleteriously altering construct properties.

\section{Acknowledgment}

Funding was provided by NIH grant R01AR053286.

\section{References}

-Adesida, A.B., A. Mulet-Sierra, L. Laouar, N.M. Jomha (2012) Oxygen tension is a determinant of the matrix-forming phenotype of cultured human meniscal fibrochondrocytes. PLoS One 7: e39339.

Adkisson, H.D., C. Milliman, X. Zhang, K. Mauch, R.T. Maziarz, P.R. Streeter (2010) Immune evasion by neocartilage-derived chondrocytes: implications for biologic repair of joint articular cartilage. Stem Cell Res 4: 57-68.

-Almarza, A.J., K.A. Athanasiou (2004) Seeding techniques and scaffolding choice for tissue engineering of the temporomandibular joint disk. Tissue Eng 10: 1787-1795.

Asanbaeva, A., K. Masuda, E.J. Thonar, S.M. Klisch, R.L. Sah (2007) Mechanisms of cartilage growth: modulation of balance between proteoglycan and collagen in vitro using chondroitinase ABC. Arthritis Rheum 56: 188-198.

Athanasiou, K.A., E.M. Darling, J.C. Hu (2009) Articular Cartilage Tissue Engineering. Synthesis Lectures on Tissue Engineering. San Rafael, Morgan \& Claypool, vol 1, pp 1-182.

Athanasiou, K.A., J. Sanchez-Adams (2009) Engineering the Knee Meniscus. Synthesis Lectures on Tissue Engineering. San Rafael, Morgan \& Claypool, pp 1-97.

Barbero, A., S. Grogan, D. Schafer, M. Heberer, P. Mainil-Varlet, I. Martin (2004) Age related changes in human articular chondrocyte yield, proliferation and post-expansion chondrogenic capacity. Osteoarthritis Cartilage 12: $476-484$.

Cheuk, Y.C., M.W. Wong, K.M. Lee, S.C. Fu (2011) Use of allogeneic scaffold-free chondrocyte pellet in repair of osteochondral defect in a rabbit model. J Orthop Res 29: 13431350.
Chia, H.N., M.L. Hull (2008) Compressive moduli of the human medial meniscus in the axial and radial directions at equilibrium and at a physiological strain rate. J Orthop Res 26: 951-956.

Darling, E.M., K.A. Athanasiou (2005) Rapid phenotypic changes in passaged articular chondrocyte subpopulations. J Orthop Res 23: 425-432.

Gan, L., R.A. Kandel (2007) In vitro cartilage tissue formation by co-culture of primary and passaged chondrocytes. Tissue Eng 13: 831842.

Giannoni, P., A. Pagano, E. Maggi, R. Arbico, N. Randazzo, M. Grandizio, R. Cancedda, B. Dozin (2005) Autologous chondrocyte implantation (ACI) for aged patients: development of the proper cell expansion conditions for possible therapeutic applications. Osteoarthritis Cartilage 13: 589-600.

Gunja, N.J., K.A. Athanasiou (2007) Passage and reversal effects on gene expression of bovine meniscal fibrochondrocytes. Arthritis Res Ther 9: R93.

Hendriks, J., J. Riesle, C.A. Vanblitterswijk (2006) Effect of stratified culture compared to confluent culture in monolayer on proliferation and differentiation of human articular chondrocytes. Tissue Eng 12: 2397-2405.

Hidaka, C., C. Cheng, D. Alexandre, M. Bhargava, P.A. Torzilli (2006) Maturational differences in superficial and deep zone articular chondrocytes. Cell Tissue Res 323: 127-135.

Hu, J.C., K.A. Athanasiou (2006) A self-assembling process in articular cartilage tissue engineering. Tissue Eng 12: 969-979.
Huey, D.J., K.A. Athanasiou (2011) Maturational growth of self-assembled, functional menisci as a result of TGF-betal and enzymatic chondroitinase- $\mathrm{ABC}$ stimulation. Biomaterials 32: 2052-2058.

Huey, D.J., J.C. Hu, K.A. Athanasiou (2012) Chondrogenically-tuned expansion enhances the cartilaginous matrix forming capabilities of primary, adult, leporine chondrocytes. Cell Transplantation, http://www.ingentaconnect. com/content/cog/ct/pre-prints/ct0545huey.

Jubel, A., J. Andermahr, G. Schiffer, J. Fischer, K.E. Rehm, M.J. Stoddart, H.J. Hauselmann (2008) Transplantation of de novo scaffoldfree cartilage implants into sheep knee chondral defects. Am J Sports Med 36: 1555-1564.

Kim, Y.J., R.L. Sah, J.Y. Doong, A.J. Grodzinsky (1988) Fluorometric assay of DNA in cartilage explants using Hoechst 33258. Anal Biochem 174: 168-176.

Lyu, S.R., W.T. Wu, C.C. Hou, W.H. Hsieh (2010) Study of cryopreservation of articular chondrocytes using the Taguchi method. Cryobiology 60: 165-176.

Malpeli, M., N. Randazzo, R. Cancedda, B. Dozin (2004) Serum-free growth medium sustains commitment of human articular chondrocyte through maintenance of Sox 9 expression. Tissue Eng 10: 145-155.

Mandl, E.W., H. Jahr, J.L. Koevoet, J.P. van Leeuwen, H. Weinans, J.A. Verhaar, G.J. van Osch (2004) Fibroblast growth factor-2 in serumfree medium is a potent mitogen and reduces dedifferentiation of human ear chondrocytes in monolayer culture. Matrix Biol 23: 231241. 
Martin, J.M., M. Smith, M. Al-Rubeai (2005) Cryopreservation and in vitro expansion of chondroprogenitor cells isolated from the superficial zone of articular cartilage. Biotechnol Prog 21: 168-177.

Mayhew, T.A., G.R. Williams, M.A. Senica, G. Kuniholm, G.C. Du Moulin (1998) Validation of a quality assurance program for autologous cultured chondrocyte implantation. Tissue Eng 4: 325-334.

Ofek, G., C.M. Revell, J.C. Hu, D.D. Allison, K.J. Grande-Allen, K.A. Athanasiou (2008) Matrix development in self-assembly of articular cartilage. PLoS One 3: e2795.
Park, K., J. Huang, F. Azar, R.L. Jin, B.H. Min, D.K. Han, K. Hasty (2006) Scaffold-free, engineered porcine cartilage construct for cartilage defect repair - in vitro and in vivo study. Artif Organs 30: 586-596.

Rendal-Vazquez, M.E., E. Maneiro-Pampin, M. Rodriguez-Cabarcos, O. Fernandez-Mallo, I. Lopez de Ullibarri, C. Andion-Nunez, F.J. Blanco (2001) Effect of cryopreservation on human articular chondrocyte viability, proliferation, and collagen expression. Cryobiology 42: $2-10$.

Seddighi, M.R., D.J. Griffon, D.J. Schaeffer, B.A. Fadl-Alla, J.A. Eurell (2008) The effect of chondrocyte cryopreservation on cartilage engineering. Vet J 178: 244-250.

-Steinberg, M.S. (2007) Differential adhesion in morphogenesis: a modern view. Curr Opin Genet Dev 17: 281-286.
Tumia, N.S., A.J. Johnstone (2004) Promoting the proliferative and synthetic activity of knee meniscal fibrochondrocytes using basic fibroblast growth factor in vitro. Am J Sports Med 32: 915-920.

Wei, X., T. Rasanen, K. Messner (1998) Maturation-related compressive properties of rabbit knee articular cartilage and volume fraction of subchondral tissue. Osteoarthritis Cartilage 6: 400-409.

Wolf, F., C. Candrian, D. Wendt, J. Farhadi, M. Heberer, I. Martin, A. Barbero (2008) Cartilage tissue engineering using pre-aggregated human articular chondrocytes. Eur Cell Mater 16: 92-99. 